\title{
Telechelic diols from polyhydroxybutyrate via alcoholysis with ethylene glycol or glycerol
}

\author{
Xue Dan ${ }^{1, a}$, Lv Wei $^{2, b}$ and Zhang Heng ${ }^{1, c}$ \\ ${ }^{1}$ School of Science, Northwestern Polytechnical University, Xi' an 710072, China; \\ ${ }^{2}$ Oil \& Gas Technology Research Institute, Changqing OilField Company,Xi' an,710018, China. \\ axdnwpu@163.com, 'Ivxsyu@163.com, 'zhang-2667@163.com
}

Keywords: Polyhydroxybutyrate, Alcoholysis, Thermal properties, Molecular weight

\begin{abstract}
Controlled degradation of poly-hydroxybutyrate (PHB) can be obtained by alcoholysis with two types of alcohol in the presence of p-toluenesulfonic acid as catalyst. PHB oligomers terminated with free hydroxyl groups were prepared in this way and characterized using NMR, DSC and $\mathrm{TG}$ analyses. The degradation proceeds by random chain scission and the molecular weight was decreased by almost two orders of magnitude. The melting temperature, $\mathrm{T}_{\mathrm{m}}$, and the degradation temperature $\left(\mathrm{T}_{\mathrm{d}}\right)$ of PHB after alcoholysis, investigated by differential scanning calorimetry (DSC) and $T G$ analyses show a decrease in $T_{m}$ and $T_{d}$ with decreased molecular weight. Oligomers terminated with free reactive hydroxyl groups and $\mathrm{T}_{\mathrm{m}}$ decreased by approximately $22^{\circ} \mathrm{C}$ are liable to be used in the next step for further modification.
\end{abstract}

\section{Introduction}

PHB is a thermoplastic polyester with mechanical properties close to those of isotactic polypropylene, which can be extruded, moulded, and spun using conventional processing equipment ${ }^{[1]}$. Therefore, PHB may be serve as an innovative type of tissue engineering scaffold or an ideal controllable drug delivery system ${ }^{[2,3]}$. However, PHB has some deficiencies that limit its wide application as a technical material: very narrow processability window and a relatively low impact resistance $^{[4]}$. These are mainly related to its relatively high melting temperature and high crystallinity. The literature concerning PHB chemical modification focuses on biosynthesis modification, reacting modification of macromonomer and radiation method ${ }^{[5,6]}$. One route seems to hamper the drawbacks of PHB is the synthesis of block copolymers based on PHB and other synthetic biodegradable component. Functional PHA have also been prepared by incorporating various functional monomers or other metabolites in the polymer sequence.Telechelic PHB of low molecular weights with hydroxyl functionalities at each ends has been functionalized by a transesterification reaction of alkanediols and high molecular weight PHB.

Herein, the synthesis of the telechelic PHB with different molecular weight was done in the presence of ethylene glycol or glycerol by catalysis of p-toluenesulfonic acid. The chemical structure, molecular weight and distribution of terminated prepolymers were characterized by ${ }^{1} \mathrm{H}$,nuclear magnetic resonance spectrum(NMR). The effect of alcoholysis time on molecular weight of product was compared. The terminal hydroxyl content was measured by the use of acetic anhydride - pyridine, which providing accurate quantitation for the further chemical modification.

\section{Experimental procedure}

Materials PHB (Mn -7.0 $\left.\times 10^{5} \pm 10 \%\right)$ was purchased from Zhejiang Tianan Tech Co. Ltd. (Zhejiang,China). All PHB samples were purified by dissolving in chloroform following by filtration and precipitated in ether before use. Chloroform, p-toluenesulfonic acid (PTSA), ethylene glycol were all from Kemiou Reagent Development Center (Tianjin, China) and used as received.

Preparation of PHB-diol prepolymer Dihydroxyl terminated prepolymers PHB-diol were prepared by transesterification between the purified PHA materials and ethylene glycol using 
p-toluenesulfonic acid as catalyst. Typically, Ten grams of PHB was dissolved in 100ml of chloroform under reflux in the three-neck flask. Chloroform solution of 10-fold molar excess of ethylene glycol or glycerol with respect to PHB with or without catalyst was added to boiling solution of polymer. p-toluenesulfonic acid (4.8g) and ethylene glycol $(20 \mathrm{~g})$ were added in subsequently. The reaction temperature was controled at $60^{\circ} \mathrm{C}$. The reaction was carried out under reflux for $2-24 \mathrm{~h}$ depending on the molecular weight of PHB-diol required. The resultant solution was washed with distilled water for 3 times, concentrated and dried under reduced pressure.

Materials' characterizations The chemical structure and monomer composition were determined by ${ }^{1} \mathrm{HNMR}$ using ADVANCEIII400MHz NMR spectrometer. The NMR spectrum was obtained at room temperature in $\mathrm{CDCl} 3(20 \mathrm{mg} / \mathrm{ml})$ with tetramethylsilane (TMS) as an internal standard. Calorimetric measurements were performed by differential scanning calorimetry using a MettlerDSC-30 instrument. The PHB specimens were scanned in nitrogen atmosphere at a heating rate of $10 \sim$ in the temperature range $25-125^{\circ} \mathrm{C}$ (the first cycle) when the melting parameters of the crystalline part of the specimen were registered. Then, the specimens were rapidly cooled $(=100 \sim$ and scanned again at a rate of $10 \sim$ in the temperature range from -30 to $220^{\circ} \mathrm{C}$ (the second cycle). Thermo-gravimetric (TGA) measurements were made with a TA-Q50 thermo-gravimetric analyzer(TA,USA), under nitrogen flow $(20 \mathrm{ml} / \mathrm{min})$ with a scan rate of $10^{\circ} \mathrm{C} / \mathrm{min}$.

Viscometry Intrinsic viscosity of solutions was measured using a Ubbelohde dilution viscometer with a curved capillary. All the measurements were done in chloroform at room temperature. The solution of each sample was prepared in such concentration that the relative viscosity of solutions prepared from the initial one by diluting was in the range from 1.1 to 1.8 . For the evaluation of the data obtained the Huggins relationship was used

$$
\frac{\eta_{s p}}{c}=[\eta]+k_{H}\left[\eta^{2}\right] c
$$

where $\eta_{\mathrm{sp}},[\eta]$, c, and $\mathrm{k}_{\mathrm{H}}$ stand for specific viscosity, limiting viscosity number, concentration, and Huggins constant, respectively.

The values of limiting viscosity number were used for determination of viscosity molecular weights according to following relation for this polymer, solvent, and temperature ${ }^{[7]}$ :

$$
[\eta]_{\mathrm{CHCl}_{3}}^{30^{\circ} \mathrm{C}}=1.18 \times 10^{-4} \mathrm{M}_{v}^{0.78}
$$

where $[\eta]_{\mathrm{CHCl}_{3}}^{30^{\circ} \mathrm{C}}$ is the intrinsic viscosity in $\mathrm{dl} / \mathrm{g}$.

\section{Results and discussion}

A series of experiments had been employed to screen a number of different esterification catalysts and degradation reactants. All reactions shared the following procedure: the starting polyester, PHB, was dissolved at a concentration of $10 \mathrm{wt} \%$ in the solvent at $60^{\circ} \mathrm{C}$ and the degradation reactant was added (sometimes in about tenfold excess). The catalyst was then admixed and the reaction was stopped by cooling. The reaction products were precipitated in water, isolated, purified, and analyzed by means of nuclear magnetic resonance (NMR). The synthetic schematic route of the controlled alcoholysis of bacterial PHB is showed in Fig. 1.

As the experimental part described, PHB prepolymer was obtained by alcoholysis of bacteria PHB. In the presence of acidic catalyst p-toluenesulfonic acid, the ethylene glycol or glycerol broke the ester bond giving rise to double-hydroxyl terminated product ${ }^{[8,9]}$. Since the alcoholysis proceeded according to a random cleavage mechanism ${ }^{[10]}$, the molecular weight of the degraded product decreased rapidly in the initial stage. As the reaction went on, the rate of decrease would reduce, and finally yellow oil products would be obtained if the reaction time reached to $24 \mathrm{~h}$.

The NMR spectra of PHB-diol are shown in Figs. $2{ }^{1} \mathrm{HNMR}\left(\mathrm{CDCl}_{3}, \mathrm{~d}, \mathrm{ppm}\right): 5.21-5.29$ (due to protons c), 4.21-4.23 (due to protons $\mathrm{f}$ and $\mathrm{h}$ ), 3.81(due to protons $\mathrm{d}$ and e), 2.41-2.66 (due to protons b), $1.22-1.32$ (due to protons a). This proved that the hydroxyl terminated PHB-diol were produced successfully. 


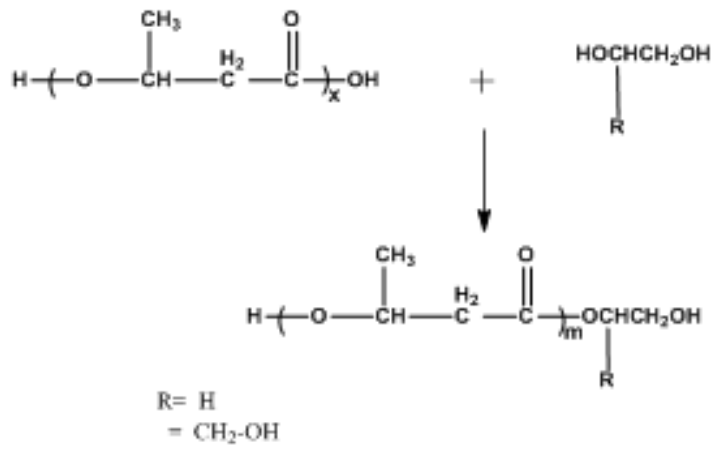

Fig. 1 Degradation reaction of PHB with ethylene glycol and glycerol.

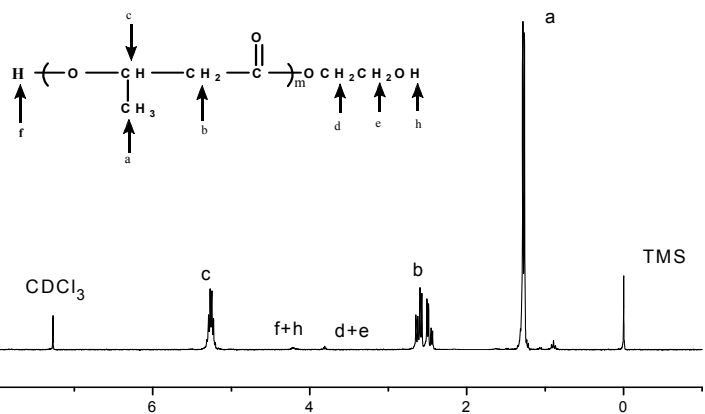

Figure. $2400 \mathrm{MHz}{ }^{1} \mathrm{H}$ NMR spectrum of PHB-diol in $\mathrm{CDCl} 3$

Effect of alcoholysis on PHB molecular weight Polymers of PHB were achieved by alcoholysis with two types of alcohol differing in the number of hydroxyl groups, which ethylene glycol and glycerol were chosen for this purpose. Results of alcoholysis with both alcohols are summarized in Table 1. The alcohol used is symbolised by EG for ethylene glycol or GLY for glycerol in the transesterification agent. Initially, p-toluene sulfonic acid was used as an acid catalyst in the reaction of PHB with ethylene glycol. The reaction had abrupt behavior and starting material PHB degraded within $2 \mathrm{~h}$ down to half of the original molecular weight. Oligomers with higher molecular weight were obtained when shorter time of alcoholysis was applied. After $4 \mathrm{~h}$, degradation by $80 \%$, was observed based on the changes of original molecular weight Mv. Reaction in the absence of catalyst was significantly slower than catalyzed reaction and the degradation of the starting material was hardly observable after $4 \mathrm{~h}$.

The next series of reactions was made with glycerol in the presence of the same catalyst (summarized in Table 1 as GLY samples). This alcoholysis is slower than the reaction with ethylene glycol. The observed difference is due to a larger spatial requirement for bulky glycerol than that of linear molecule of ethyl eneglycol, which results in a reduction of relative accessibility of reaction centres of polyester and alcohol.

Thermal properties The thermal transitions and thermal stability of degraded PHB specimens were evaluated by DSC and TGA respectively. This method serves as convenient tool for estimation of PHB degradation ${ }^{[1]}$. The typical DSC thermograms of selected degraded PHB samples are shown in Fig. 3. It is seen that $\mathrm{T}_{\mathrm{m}}$ decreased by approximately $22^{\circ} \mathrm{C}$ with the molecular weight down to $\mathrm{Mv}=5,000 \mathrm{~g} / \mathrm{mol}$. Thermal stability of the degraded PHB was evaluated using TGA under dynamic $\mathrm{N}_{2}$ atmosphere. Fig.4 shows the thermal degradation profile of PHB-diol, along with those of its PHB precursor. Temperature at which $10 \%$ weight loss has occurred is taken as the degradation temperature $\left(\mathrm{T}_{\mathrm{d}}\right)$ of each block ${ }^{[12]}$. The values of PHB-diol is $227{ }^{\circ} \mathrm{C}$ and that of PHB is $270^{\circ} \mathrm{C}$. Thermal stability of PHB-diol decreased compared with that of precursor, which is mainly due to the alcoholysis reaction significantly reduction of the molecular weight of PHB.

\section{Summary}

A controlled degradation of PHB by alcoholysis in the presence of p-toluene sulfonic acid as a catalyst was performed. Different oligomers terminated with free reactive hydroxyl groups were prepared, which can be used in the next step for crosslinking and chain-extension reactions. Alcoholysis with ethylene glycol is significantly faster than with glycerol and the results show that degradation proceeds by random chain scission. The molecular weight was decreased by almost two orders of magnitude depending on the alcoholysis conditions. $\mathrm{T}_{\mathrm{m}}$ and $\mathrm{T}_{\mathrm{d}}$ of PHB after alcoholysis, show a decrease with decreased molecular weight. 
Table 1 Average molecular weight of PHB polymers obtained by viscometry in chloroform (Visc.)

\begin{tabular}{c|c|c|c}
\hline Transesterification agent & Catalyst & Reaction time/h & Visc. Mv \\
\hline- & - & 0 & 600000 \\
EG & PTSA & 2 & 306000 \\
EG & PTSA & 4 & 123000 \\
EG & PTSA & 6 & 23000 \\
EG & - & 6 & 586000 \\
EG & PTSA & 10 & 20135 \\
EG & PTSA & 12 & 17000 \\
EG & PTSA & 14 & 11030 \\
EG & PTSA & 16 & 8000 \\
EG & PTSA & 18 & 7010 \\
EG & PTSA & 20 & 6820 \\
EG & PTSA & 24 & 5230 \\
EG & PTSA & 4 & 4890 \\
GLY & PTSA & 6 & 284600 \\
GLY & PTSA & 8 & 113000 \\
GLY & PTSA & 12 & 49630 \\
GLY & PTSA & & 29360 \\
\hline
\end{tabular}

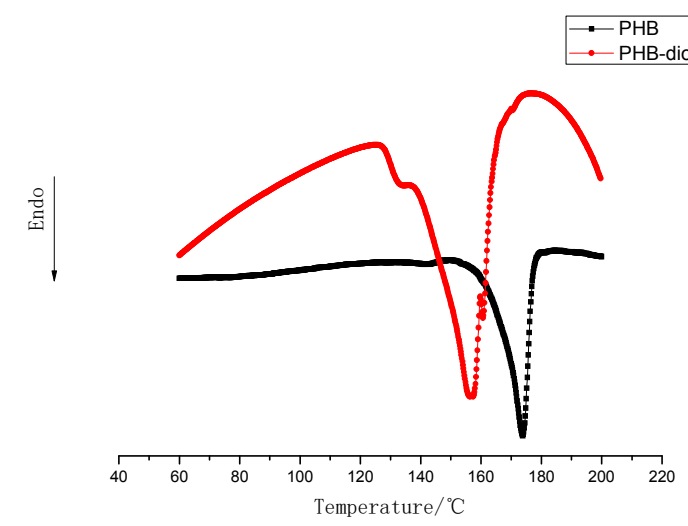

Fig. 3 DSC records for samples of PHB and PHB-diol prepared by alcoholysis with ethylene glycol.

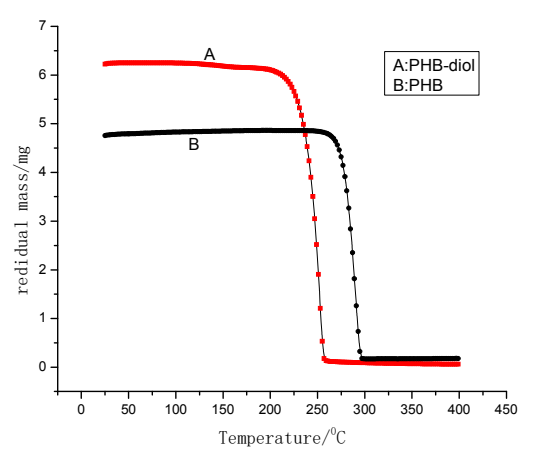

Fig. 4 Thermal degradation profiles of PHB and PHB-diol prepared by alcoholysis with ethylene glycol.

\section{Acknowledgments}

We are grateful to Education special research Foundation of Shaanxi for the financial support. (Project No. 14JK1569). Xue Dan is corresponding author of this paper.

\section{References}

[1] Xu Li, Xian Jun Loh, Ke Wang, Poly(ester urethane)s Consisting of Poly[(R)-3-hydroxybutyrate] and Poly(ethylene glycol) as Candidate Biomaterials:Characterization and Mechanical Property Study, Biomacromolecules, 6 (2005) 2740-2747.

[2] Khanna S, Srivastava AK., Recent advances in microbial polyhydroxyalkanoates. Progress Biochem. 40(2005) 607-619.

[3] Kerh Li Liu, Eugene Shi Guang Choo, Siew Yee Wong, Designing Poly[(R)-3- hydroxybutyrate] -Based Polyurethane Block Copolymers for Electrospun Nanofiber Scaffolds with Improved Mechanical Properties and Enhanced Mineralization Capability, J. Phys. Chem. B, 114(2010) 7489-7498. 
[4] H.F. Naguib, M.S. Abdel Aziz, G.R. Saad, Synthesis, morphology and thermal properties of polyurethanes nanocomposites based on poly(3-hydroxybutyrate) and organoclay, Journal of Industrial and Engineering Chemistry 19 (2013) 56-62.

[5] Qiang Zhao, Guoxiang Cheng, Haiming Li, Synthesis and characterization of biodegradable poly(3-hydroxybutyrate) and poly(ethylene glycol) multiblock copolymers, Polymer 46 (2005) 10561-10567.

[6] Kerh Li Liu, Suat Hong Goh, Jun Li, Controlled synthesis and characterizations of amphiphilic poly[(R,S )-3-hydroxybutyrate]-poly(ethylene glycol)-poly[(R,S )-3-hydroxybutyrate] triblock copolymers, Polymer 49 (2008) 732-741.

[7] Zdeno S` pitalsky', Igor Lacı'k, Elena Lathova', Controlled degradation of polyhydroxybutyrate via alcoholysis with ethylene glycol or glycerol, Polymer Degradation and Stability 91(2006) 856-861.

[8] Yamanaka Kenji, Kimura Yoshiharu1, Aoki Takashi, Effect of ethylene glycol on the end group structure of poly(3-hydroxybutyrate), Polymer Degradation and Stability,95(2010) 1284-1291.

[9] Zhao Q, Cheng GX. Preparation of biodegradable poly(3-hydroxybutyrate) and poly(ethylene glycol) multiblock copolymers. J Mater Sci, 39 (2004) 3829-3831.

[10] Thomas D. Hirt, Peter Neuenschwander, Ulrich W Suter, Telechelic diols from poly[(R)-3hydroxybutyric acid] and poly $\{[(\mathrm{R})-3$-hydroxybutyric acid]-co-[(R)-3-hydroxyvaleric acid $]\}$, Macromol. Chem. Phys., 197(1996) 1609-1614.

[11] Janigová, Ivica1, Lacík, Igor1, Chodák, Ivan, Thermal degradation of plasticized poly(3-hydroxybutyrate) investigated by DSC, Polymer Degradation and Stability, 77(2002) 35-41.

[12] Arnal,M.L., Balsamo, V., López-Carrasquero, F.,Synthesis and characterization of polystyreneb- poly(ethylene oxide)-b-poly( $\varepsilon$-caprolactone) block copolymers, Macromolecules, 23 (2001) 7973-7982. 